\title{
Quantifying entanglement of a two-qubit system via measurable and invariant moments of its partially transposed density matrix
}

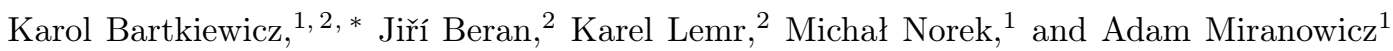 \\ ${ }^{1}$ Faculty of Physics, Adam Mickiewicz University, PL-61-614 Poznań, Poland \\ ${ }^{2}$ RCPTM, Joint Laboratory of Optics of Palacky University and \\ Institute of Physics of Academy of Sciences of the Czech Republic, \\ 17. listopadu 12, 772 07 Olomouc, Czech Republic
}

(Dated: October 2, 2018)

\begin{abstract}
We describe a direct method to determine the negativity of an arbitrary two-qubit state in experiments. The method is derived by analyzing the relation between the purity, negativity, and a universal entanglement witness for two-qubit entanglement. We show how the negativity of a twoqubit state can be calculated from just three experimentally-accessible moments of the partiallytransposed density matrix of a two-photon state. Moreover, we show that the negativity can be given as a function of only six invariants, which are linear combinations of nine invariants from the complete set of 21 fundamental and independent two-qubit invariants. We analyze the relation between these moments and the concurrence for some classes of two-qubit states (including the $X$ states, as well as pure states affected by the amplitude-damping and phase-damping channels). We also discuss the possibility of using the universal entanglement witness as an entanglement measure for various classes of two-qubit states. Moreover, we analyze how noise affects the estimation of entanglement via this witness.
\end{abstract}

PACS numbers: 03.67.Mn, 42.50.Dv

\section{INTRODUCTION}

Quantum entanglement [1, 2], which is an intrinsically and fundamentally nonclassical effect, has attracted an enormous number of works related to quantum information processing and quantum engineering in the last two decades (for reviews see, e.g., Refs. [3 [5]). Although, our understanding of quantum entanglement is much deeper now, there are still many open fundamental problems as listed, e.g., in Ref. [6]. Some of these problems address the question how to experimentally detect and estimate entanglement of a given state [7].

One could think that the most natural and simplest way to measure the entanglement of an unknown state of $\rho$ is to apply quantum state tomography (QST). This approach enables the reconstruction of $\rho$ by postprocessing experimental data, and, then, the calculation of arbitrary entanglement measures for $\rho$. Indeed, various effective QST methods have been developed [8], including those for the reconstruction of the polarization states of two photons (for a recent comparison see Ref. [9]). Nevertheless, this complete reconstruction requires to measure also a large number of parameters, which are irrelevant for the determination of entanglement. This number scales with the square of the dimension of a measured state $\rho$. Moreover, QST based on linear inversion often leads to unphysical reconstructed states. Then, nonlinear methods (based, e.g., on maximum-likelihood estimation) have to be applied to overcome this problem.

Thus, usually, entanglement is detected and quantified

*Electronic address: bark@amu.edu.pl by measuring entanglement witnesses [10] (for a review see Ref. [4]). This approach corresponds to testing the violations of classical inequalities. The operational usefulness of entanglement witnesses has been demonstrated in numerous experimental (see, e.g., earlier experiments reported in Refs. [11-14]) and theoretical works. The latter include approaches based on: polynomial moments [15] 17, collective entanglement witnesses [16, 18, 20, experimental adaptive witnesses [21, 22], and others (see, e.g., Refs. [23 27].

Some of such studies of entanglement witnesses were focused on the quantitative description of entanglement (see, e.g., recent Refs. 28 37] and a review [7 for older references). For example, a lower bound on a generic entanglement measure can be derived from the mean value of entanglement witnesses based on the Legendre transform 38 40. The estimation of the concurrence and/or negativity from entanglement witnesses was studied in, e.g., Refs. [30, 41-47. In particular, the violation of a Bell inequality, which is also an entanglement witness, can be used to estimate the concurrence [48, the negativity [49, or the relative entropy of entanglement (REE) [50]. A related problem is the estimation of one entanglement measure from another entanglement measure, e.g., the concurrence from negativity [45, 51, 52] or the REE [53, the negativity from the REE [54], or vice versa.

These approaches based on entanglement witnesses are useful and efficient, but still their usage is limited, because some information about the state should be known prior to its measurement.

In this paper, we study a universal entanglement witness (UWE), which can be used as a sufficient and necessary test of the entanglement of a two-qubit system. 
The UWE is defined as the determinant of a partiallytransformed density matrix, $\operatorname{det} \hat{\rho}^{\Gamma}$ 4. This witness can be given as a function of the moments $\Pi_{n}=\operatorname{tr}\left[\left(\hat{\rho}^{\Gamma}\right)^{n}\right]$ [55], which are directly measurable, as recently described in Ref. [56] for a linear-optical setup. The proposed setup is based on the experimental methods described and referenced in Refs. [57 [59].

Here we address the problem of applying the UWE to quantify two-qubit entanglement. Namely, the question is whether the UWE (or more precisely, its negative expectation value) can be considered a good entanglement measure. We will show that this is not the case for arbitrary two-qubit states. However, we will identify various classes of states for which the UWE is indeed a good entanglement measure. Moreover, as one of the main results of this paper, we will demonstrate that the negativity can be given as a function of the experimentallyaccessible moments $\Pi_{n}$. We will also discuss how the imperfect measurements of $\Pi_{n}$ deteriorate the estimation of the negativity.

This paper is organized as follows: In Sec. II, the UWE is defined via experimentally-accessible moments $\Pi_{n}$ of a given partially-transposed density matrix. We also show the relation between the entanglement witness and Makhlin's invariants. In Sec. III, we present one of the main results of our paper, which is the explicit formula of the negativity as a function of the experimentallyaccessible moments $\Pi_{n}$. In Sec. IV, we demonstrate when the UWE can be considered a useful entanglement measure. In Sec. V, we show the relation between the entanglement witness and the concurrence for an important class of two-qubit states, namely, the $X$-states. Our results are summarized in Sec. VI, as well as Tables I and II.

\section{UNIVERSAL ENTANGLEMENT WITNESS AND MAKHLIN INVARIANTS}

Arguably, the simplest two-qubit separability condition (Peres-Horodecki separability criterion [10, 60]) can be formulated as follows [4]: A two-qubit state $\hat{\rho}$ is entangled if and only if $\operatorname{det} \hat{\rho}^{\Gamma}<0$, where $\hat{\rho}^{\Gamma}$ is the partiallytransposed (marked by $\Gamma$ ) matrix $\hat{\rho}$. This theorem can be easily shown by recalling that the partially-transposed matrix of an arbitrary entangled two-qubit state has full rank and has exactly one negative eigenvalue. Thus, one can introduce the UWE $\hat{W}$ for a two-qubit state $\hat{\rho}$ defined as an operator for which the expected value is equal to $\operatorname{det} \hat{\rho}^{\Gamma}$. This witness can also be given in terms of the experimentally-accessible moments $\Pi_{n}=\operatorname{tr}\left[\left(\hat{\rho}^{\Gamma}\right)^{n}\right]$ as follows [55]:

$$
\begin{aligned}
W & \equiv \operatorname{det} \hat{\rho}^{\Gamma}=\langle\hat{W}\rangle:=\operatorname{tr}\left(\hat{W} \hat{\rho}^{\otimes 4}\right) \\
& =\frac{1}{24}\left(1-6 \Pi_{4}+8 \Pi_{3}+3 \Pi_{2}^{2}-6 \Pi_{2}\right) .
\end{aligned}
$$

For convenience, we call the UWE not only the observable $\hat{W}$ but also its expectation value $W$. (This convention is also used in, e.g., Ref. 61 and references therein).
In order to directly measure the UWE one could perform joint measurements on the four copies $\hat{\rho}^{\otimes 4}$ of a two-qubit state $\hat{\rho}[55]$. A direct and efficient method for the measurement of $\langle\hat{W}\rangle$ has been recently proposed for polarization qubits in a linear optical setup [56]. The witness $\hat{W}$, contrary to a typical entanglement witness, is invariant under local unitary operations, which follows from the invariance of the moments of the partially-transposed density matrix that forms the witness. This invariance is a key requirement of a good entanglement measure (see Sec. IV and, e.g., Ref. 62]).

It is worth stressing that the moments $\Pi_{n}$, in Eq. (1) for $n=2,3,4$, are not independent. To show the connection between these moments, let us analyze them in terms of the correlation matrix $\hat{\beta}$, with elements $\beta_{i j}=$ $\operatorname{tr}\left[\left(\hat{\sigma}_{i} \otimes \hat{\sigma}_{j}\right) \hat{\rho}\right]$, and the Bloch vectors $\mathbf{s}$ and $\mathbf{p}$, with elements $s_{i}=\operatorname{tr}\left[\left(\hat{\sigma}_{i} \otimes \hat{\sigma}_{0}\right) \hat{\rho}\right]$ and $p_{j}=\operatorname{tr}\left[\left(\hat{\sigma}_{0} \otimes \hat{\sigma}_{j}\right) \hat{\rho}\right]$. The matrices $\hat{\sigma}_{i}$ for $i=1,2,3$ are the Pauli matrices, and $\hat{\sigma}_{0}$ is the single-qubit identity matrix. As shown in Ref. [56], we can write the first four moments as

$$
\begin{aligned}
\Pi_{1} & =1, \\
4 \Pi_{2} & =1+x_{1}, \\
16 \Pi_{3} & =1+3 x_{1}+6 x_{2}, \\
64 \Pi_{4} & =1+6 x_{1}+24 x_{2}+x_{1}^{2}+2 x_{3}+4 x_{4},
\end{aligned}
$$

where

$$
\begin{array}{ll}
x_{1}=I_{2}+I_{4}+I_{7}, & x_{2}=I_{1}+I_{12}, \\
x_{3}=I_{2}^{2}-I_{3}, & x_{4}=I_{5}+I_{8}+I_{14}+I_{4} I_{7},
\end{array}
$$

are the functions of 9 out of the 18 Makhlin invariants [63], i.e., $I_{1}=\operatorname{det} \hat{\beta}, I_{2}=\operatorname{tr}\left(\hat{\beta}^{T} \hat{\beta}\right), I_{3}=\operatorname{tr}\left(\hat{\beta}^{T} \hat{\beta}\right)^{2}$, $I_{4}=\mathbf{s}^{2}, I_{5}=[\mathbf{s} \hat{\beta}]^{2}, I_{7}=\mathbf{p}^{2}, I_{8}=[\hat{\beta} \mathbf{p}]^{2}, I_{12}=\mathbf{s} \hat{\beta} \mathbf{p}$, and $I_{14}=e_{i j k} e_{l m n} s_{i} p_{l} \beta_{j m} \beta_{k n}$, where $e_{i j k}$ is the Levi-Civita symbol. The invariants are also a subset of 21 fundamental and independent two-qubit invariants described by King and Welsh in Ref. 64]. This demonstrates explicitly that, in general, in order to measure the UWE one needs to measure these nine fundamental physical quantities (invariants). Any function of invariants is also an invariant. We can, therefore, introduce the following six independent invariants that need to be measured to estimate the values of moments $\Pi_{n}$ for $n=2,3,4$. These invariants are

$$
\begin{aligned}
& y_{1}=I_{2}, \quad y_{2}=I_{4}, \quad y_{3}=I_{7}, \quad y_{4}=I_{1}+I_{12}, \\
& y_{5}=I_{5}+I_{8}+I_{14}, \quad y_{6}=I_{3} .
\end{aligned}
$$

This means that in order to quantify entanglement via the UWE, one needs to measure exactly six instead of nine independent quantities. The number of necessary measurements is by 10 smaller than the number of measurements needed for a full quantum-state tomography. We can conjecture that this is the minimum number of independent measurements needed for estimating the entanglement of an arbitrary two-qubit state. 


\section{NEGATIVITY VIA MOMENTS OF $\hat{\rho}^{\Gamma}$}

In order to quantify the Peres-Horodecki separability criterion [10, 60], Życzkowski et al. 65] introduced a parameter later referred to as negativity. Subsequently, Vidal and Werner [66] proved that the negativity is an entanglement monotone, so can be used as an entanglement measure. The negativity has an operational meaning as the entanglement cost under operations preserving the positivity of partial transpose (PPT) [67, 68. It can also be used as an estimator of entangled dimensions, i.e., to estimate the number of entangled degrees of freedom of two subsystems [69]. The negativity of a two-qubit state $\hat{\rho}$ is usually defined as

$$
N=2 \max \left\{0,-\min \left[\operatorname{eig}\left(\hat{\rho}^{\Gamma}\right)\right]\right\},
$$

in terms of the minimum (negative) eigenvalue $\lambda \equiv-\mu=$ $\min \left[\operatorname{eig}\left(\hat{\rho}^{\Gamma}\right)\right](\mu>0)$ of the partially-transposed density matrix $\hat{\rho}^{\Gamma}$. The task of finding $\lambda$ is usually not easy because the operation of partial transposition is not physical, so this operator can only be implemented approximately.

There is another approach based on measuring moments $\Pi_{n}=\operatorname{tr}\left(\hat{\rho}^{\Gamma}\right)^{n}$ of the partially-transposed matrix $\hat{\rho}^{\Gamma}$ of a two-qubit state $\hat{\rho}$. It has recently been shown in Ref. [56] that all four first moments $\Pi_{n}$ can be measured directly using at most four copies of the investigated two-qubit state. This was shown on the example of the measurement of a two-photon polarization state by using a linear-optical setup. We note that this approach of Ref. [56] can be generalized to other implementations of qubits and various setups.

The first two moments $\Pi_{n}$ are equivalent to the trace and purity of $\hat{\rho}$, i.e., $\Pi_{1}=1$ and $\Pi_{2}=p$ respectively. An efficient method for measuring the purity of an arbitrary polarization state of two photons has been proposed recently in Ref. [59]. The higher-order moments $\Pi_{3}$ and $\Pi_{4}$ can be measured as described in Ref. [56]. Let us also mention that, as long as there is some entanglement, $\hat{\rho}^{\Gamma}$ has four nonzero eigenvalues, among which only one is negative and equals to $\lambda$. This property holds for an arbitrary two-qubit state [70.

Let us derive an expression for the negativity in terms of the experimentally-accessible moments $\Pi_{n}$ for $n=1,2,3$. The principal invariants of the partiallytransposed density matrix read

$$
\begin{aligned}
J_{1} & =\Pi_{1}=\operatorname{tr} \hat{\rho} \hat{\rho}^{\Gamma}=\lambda_{1}+\lambda_{2}+\lambda_{3}-\mu=1, \\
J_{2} & =\frac{1}{2}\left(\Pi_{1}^{2}-\Pi_{2}\right)=\frac{1}{2}\left[\left(\operatorname{tr} \hat{\rho}^{\Gamma}\right)^{2}-\operatorname{tr}\left(\hat{\rho}^{\Gamma}\right)^{2}\right] \\
& =\lambda_{1} \lambda_{2}+\lambda_{2} \lambda_{3}+\lambda_{3} \lambda_{1}-\mu(1+\mu), \\
J_{3} & =\operatorname{det} \hat{\rho}^{\Gamma}=-\lambda_{1} \lambda_{2} \lambda_{3} \mu,
\end{aligned}
$$

where $\lambda_{n}$ for $n=1,2,3$ are the positive eigenvalues $\rho^{\Gamma}$ and $\mu$ is the module of the negative eigenvalue. After simple algebraic manipulations we derive

$$
\begin{aligned}
\sum_{n=1}^{3}[ & -\lambda_{n} \mu^{3}+\frac{1}{2} \Pi_{2} \lambda_{n} \mu+\left(\lambda_{n}^{2}-\lambda_{n}\right) \mu^{2} \\
& \left.-\frac{1}{2}\left(2 \lambda_{n}^{3}-2 \lambda_{n}^{2}+\lambda_{n}\right) \mu-\operatorname{det} \hat{\rho}^{\Gamma}\right]=0 .
\end{aligned}
$$

This sum can be directly calculated using the definition of moments $\Pi_{n}$. As a result we obtain the following expression

$$
\begin{aligned}
-3 \mu^{4}-3 \mu^{3}+\frac{3}{2}\left(\mu^{2}+\mu\right) \Pi_{2}-\Pi_{3} \mu-\frac{3}{2} \mu^{2} & \\
-3 \operatorname{det} \hat{\rho} \Gamma-\frac{1}{2} \mu & =0 .(10)
\end{aligned}
$$

Equation (10) has an important consequence, i.e., we can calculate the negativity $N$ after measuring $\Pi_{n}$ for $n=$ $1,2,3,4$. As discussed in Ref. [56] measuring these four experimentally-accessible moments can be done more efficiently than performing full quantum state tomography. Equation (10) is a fourth degree polynomial in $N=2 \mu$, which after simplification reads

$$
\begin{aligned}
48 \operatorname{det} \hat{\rho}^{\Gamma}+3 N^{4}+6 N^{3}-6 N^{2}\left(\Pi_{2}-1\right) & \\
-4 N\left(3 \Pi_{2}-2 \Pi_{3}-1\right) & =0 .
\end{aligned}
$$

In our opinion this is one of the main results of this work. We can be sure that it has solutions if $\operatorname{det} \hat{\rho}^{\Gamma}<0$ (i.e., when the state $\hat{\rho}$ is entangled). The solutions can be found analytically by applying the well-known Ferrari and Cardano formulas. Equation (11) has four solutions, however there is only one real solution where $N>0$. Therefore, the value of the negativity is uniquely defined by Eq. (11). We do not give these solutions explicitly, as they are lengthy and can be easily obtained by using a computer algebra system.

Unfortunately, the value of the negativity calculated from Eq. (11) is very sensitive to the uncertainty of measuring $\Pi_{n}$. This can be observed in Fig. 11, where the relation between the theoretical and the experimentally measured values of the negativity is depicted for several values of the maximal relative uncertainties in estimating $\Pi_{n}$. Figure 1(b) suggests that if the relative error is close to $1 \%$, the noise level starts to be too high for estimating the negativity with a reasonable precision in its entire range. For uncertainty levels $\gtrsim 10 \%$, the measurement method is not reliable for any value of $N$. Note that, in all cases, the level of the uncertainty in estimating the negativity is the largest for the values of $N \approx 0$.

\section{UNIVERSAL ENTANGLEMENT WITNESS AS AN ENTANGLEMENT MEASURE}

As shown in Fig. 2 , measuring $\hat{W}$ is less prone to noise than estimating the negativity.

It is convenient to use the rescaled value of $\langle\hat{W}\rangle$ defined as

$$
w:=\max [0,-16\langle\hat{W}\rangle] .
$$


(a)

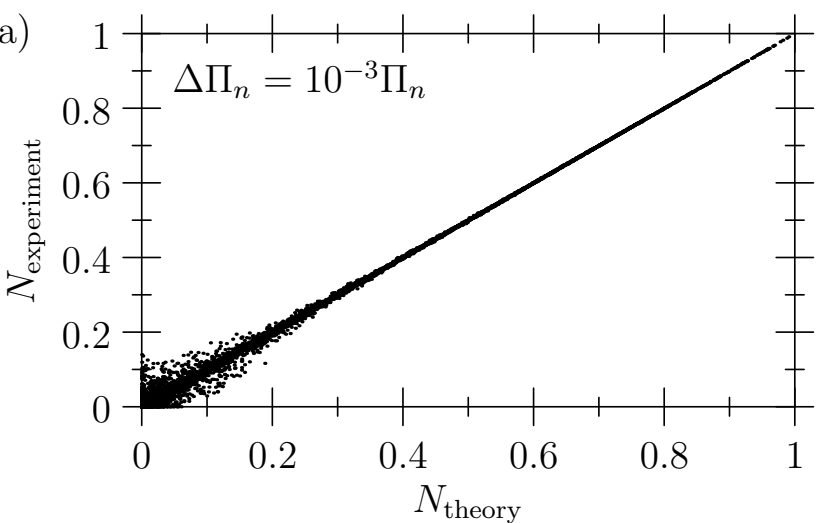

(b)

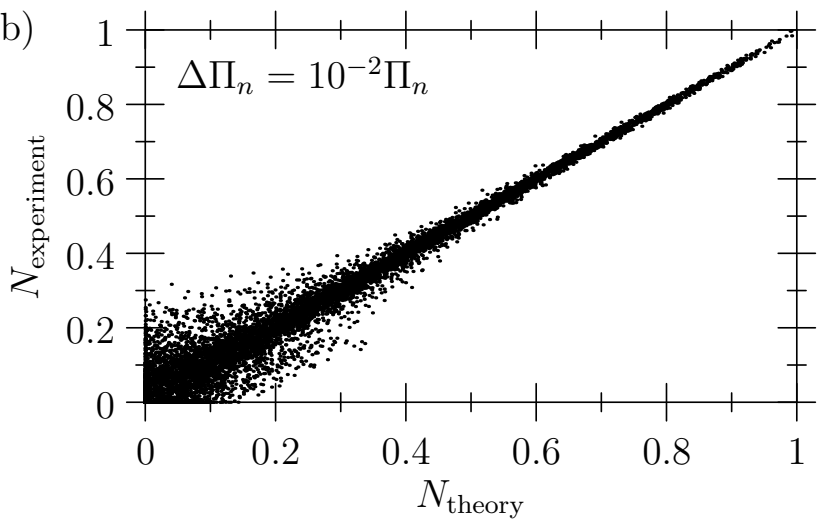

FIG. 1: The relation between the theoretical precise values, $N_{\text {theory }}$, and "experimental" noisy values, $N_{\text {experiment }}$, of the negativity for $10^{4}$ density matrices randomly generated in a Monte-Carlo simulation. For each density matrix, all these moments $\Pi_{n}$ were calculated and then a random noise $\delta \Pi_{n} \in\left[0, \Delta \Pi_{n}\right]$ was added. The maximal noise $\Delta \Pi_{n}$ was set to (a) $10^{-3} \Pi_{n}$ and (b) $10^{-2} \Pi_{n}$. This figure demonstrates that the value of the experimentally-estimated negativity is very sensitive to noise, especially if this value is approaching 0 .

Now we explicitly describe that the UWE $w$ satisfies the following standard criteria for a good entanglement measure (as listed in, e.g., Ref. [4):

C1. The inequalities hold $0 \leq w(\hat{\rho}) \leq 1$, where $w(\hat{\rho})=0$ for any unentangled state and $w(\hat{\rho})=1$ for the Bell states.

C2. Any local unitary transformations of the form $U_{A} \otimes$ $U_{B}$ do not change $w(\hat{\rho})$ for any state $\hat{\rho}$.

C3. An additional property: The witness $w(|\psi\rangle)$ is simply related to the entropy of entanglement for any pure state $|\psi\rangle$, i.e., by a relation corresponding to the Wootters formula for the entanglement of formation [71,

$$
E_{F}(w)=h\left(\frac{1}{2}[1+\sqrt{1-\sqrt{w}}]\right)
$$

where $h(y)=-y \log _{2} y-(1-y) \log _{2}(1-y)$ is binary entropy. This property follows from the observation (a)

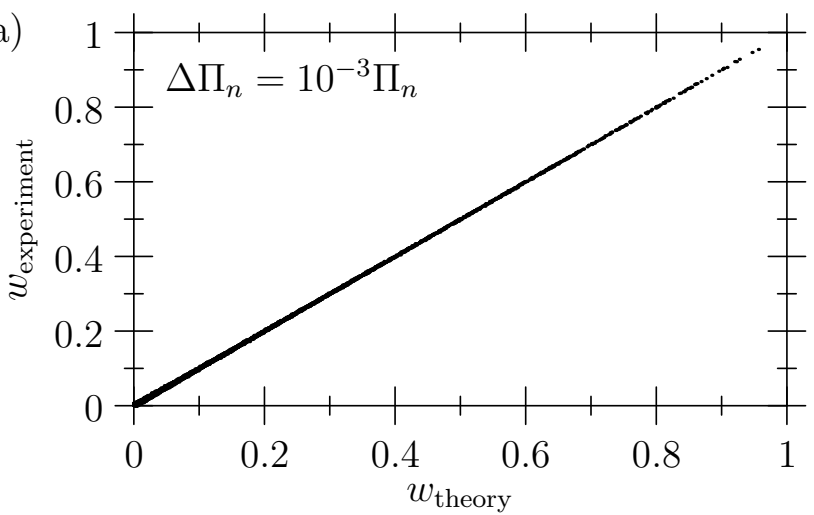

(b)

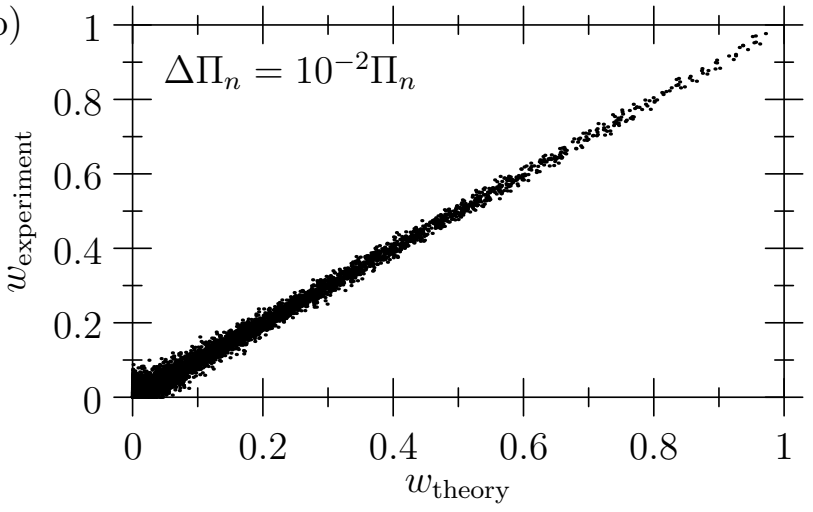

FIG. 2: Same as in Fig. 1 but for the relation between the theoretical $\left(w_{\text {theory }}\right)$ and experimental ( $\left.w_{\text {experiment }}\right)$ values of the UWE $w$, given in Eq. (12). This figure demonstrates how the value of the experimentally-estimated witness is sensitive to noise.

that

$$
w(|\psi\rangle)=N^{4}(|\psi\rangle)=C^{4}(|\psi\rangle)
$$

where $w(|\psi\rangle), N(|\psi\rangle)$, and concurrence $C(|\psi\rangle)$ are defined by Eqs. (12), (5), and 222, respectively, where $\rho$ is replaced by $|\psi\rangle\langle\psi|$. This corresponds to case 1 in Table I.

Unfortunately, in general, the following two important properties do not hold for the witness $w(\hat{\rho})$.

C4. A good entanglement measure $E(\hat{\rho})$ should not increase for any state $\hat{\rho}$ and any local operations with classical communication (LOCC). This property can be violated for $w(\hat{\rho})$ as shown in Appendix B.

C5. A good entanglement measure $E(\hat{\rho})$ should be convex under discarding information, i.e., $\sum_{i} p_{i} E\left(\hat{\rho}_{i}\right) \geq E\left(\sum_{i} p_{i} \hat{\rho}_{i}\right)$. In other words, one cannot increase $E(\hat{\rho})$ by mixing states $\hat{\rho}_{i}$. An example of the violation of this property for $w(\hat{\rho})$ is given in Appendix C.

Property C.2 follows from the fact that the UWE can be expressed as a function of local polynomial invariants 63. For pure states, the UWE is equivalent to 
the so-called $G$ concurrence [55, 72], which is a monotone under LOCC (C.3). Thus, even if the properties C.4 and C.5 are not satisfied in general, the witness $w$ for two-qubit states is a useful parameter for quantifying entanglement.

Moreover, the UWE $w$ provides tight upper and lower bounds for the negativity $N(\hat{\rho})$ of an arbitrary two-qubit state $\hat{\rho}$ [55]:

$$
f(w) \leq N \leq \sqrt[4]{w}
$$

where $f(w)$ is the inverse of the polynomial $w(N)=$ $N(N+2)^{3} / 27$ on the interval $N \in[0,1]$. Explicitly, the lower bound is given by [56],

$$
f(w)=\frac{1}{2}\left(-3+\sqrt{z}+\sqrt{3-z+\frac{2}{\sqrt{z}}}\right),
$$

where $z=1-y+x, y=36 w / x$, and

$$
x=3 \sqrt[3]{2 \sqrt{w^{2}(16 w+1)}-2 w} .
$$

We show in Table 1 that the states saturating the upper and lower bounds are pure (case 1) and Werner's states (case 8) [73], respectively.

The boundary states can be found in the set of the so-called $X$ states. These states can be simply manipulated [50] and are universal in the sense that an arbitrary two-qubit state can be converted, by a unitary transformation, into its $X$-state counterpart [74]. Moreover, the $X$ states appear as solutions in many simple physical models in, e.g., the $X Y Z$ Heisenberg model [75, 76] or decaying entangled qubits coupled to a common reservoir exhibiting the effects of sudden death 77 and rebirth 78 of entanglement. The name of these states becomes clear when its density matrix $\hat{\rho}$ is given explicitly in the standard computational basis, i.e.,

$$
\hat{\rho}=\left(\begin{array}{cc|cc}
a & 0 & 0 & b \\
0 & c & d & 0 \\
\hline 0 & d^{*} & e & 0 \\
b^{*} & 0 & 0 & f
\end{array}\right) .
$$

The partial transpose with respect to the second subsystem of two-qubit density matrix $\hat{\rho}$ reads

$$
\hat{\rho}^{\Gamma}=\left(\begin{array}{cc|cc}
a & 0 & 0 & d \\
0 & c & b & 0 \\
\hline 0 & b^{*} & e & 0 \\
d^{*} & 0 & 0 & f
\end{array}\right) .
$$

Now, it follows from the Laplace expansion that the UWE for the $X$ states can be given as a product of determinants,

$$
\begin{aligned}
W & =\operatorname{det}\left(\begin{array}{cc}
a & d \\
d^{*} & f
\end{array}\right) \operatorname{det}\left(\begin{array}{cc}
c & b \\
b^{*} & e
\end{array}\right) \\
& =\operatorname{det}\left(\begin{array}{cc}
a & |d| \\
|d| & f
\end{array}\right) \operatorname{det}\left(\begin{array}{cc}
c & |b| \\
|b| & e
\end{array}\right) .
\end{aligned}
$$

This is a four-dimensional volume (a product of two areas). We can expand it further to obtain

$$
W=[(|d|-\sqrt{a f})(|d|+\sqrt{a f})][(|b|-\sqrt{c e})(|b|+\sqrt{c e})] .
$$

This corresponds to the volume of a four-dimensional box. Note that the length of its longest negative edge (the longest edge of negative orientation) corresponds to the negativity. However, the expression for the negativity is not simple because it requires finding the smallest eigenvalue of $\hat{\rho}^{\Gamma}$, i.e., factorizing $\operatorname{det} \hat{\rho}^{\Gamma}$ in another way.

\section{UNIVERSAL ENTANGLEMENT WITNESS AND CONCURRENCE}

Remarkably, the largest negative factor in the expression for the UWE, given by Eq. (21), for the $X$ states, corresponds to another popular entanglement measure. Namely, the Wootters concurrence [71]:

$$
C(\hat{\rho})=\max \left(0,2 \lambda_{\max }-\sum_{j} \lambda_{j}\right)
$$

where $\lambda_{j}^{2}=\operatorname{eig}\left[\hat{\rho}\left(\hat{\sigma}_{2} \otimes \hat{\sigma}_{2}\right) \hat{\rho}^{*}\left(\hat{\rho}_{2} \otimes \hat{\sigma}_{2}\right)\right]_{j}$ and $\lambda_{\max }=$ $\max _{j} \lambda_{j}$. The witness $W$ can be interpreted as a geometric mean of all the lengths in Eq. 211). Thus, the UWE is not a good measure of entanglement, because it underestimates the available entanglement. However, the UWE can be used as a measure of entanglement if all the edges have the same length and the volume is negative. Additional information about the relation between the UWE and concurrence for two-qubit states can be found in Ref. [79].

Let us note that there are some constrains on the matrix elements of the $X$ states, e.g., the trace of the partially-transposed matrix equals 1 . From this observation follows that $a+c+e+f=1$. Other constrains are imposed by the fact that $\hat{\rho}$ is positive semidefinite, i.e., $|d| \leq \sqrt{c e}$ and $|b| \leq \sqrt{a f}$. By recalling some properties of density matrices, we can deduce that the UWE is a monotonic function of a proper entanglement measure, i.e., the concurrence. The concurrence is given by the following simple expression for $X$ states [80]

$$
C=2 \max (0,|d|-\sqrt{a f},|b|-\sqrt{c e}) .
$$

One can see that the UWE is related to both the negativity and concurrence for the whole class of the $X$ states. For some subclasses of the $X$ states, the negativity and concurrence are equivalent. This happens for pure states, rank-2 Bell-diagonal states, phase-damped states, Bell states with isotropic noise (i.e., the Werner [73] and Werner-like [81] states) (see cases 1, 2,3, and 8 in TableI. respectively). For the amplitude-damped states (case 4 in Table I) with the damping parameter $p=1-f$, the relation is also simple as $C=\sqrt{N^{2}+2 f N}$ [50], although it also involves the damping parameter $p$. 
In Table I] we present a survey of the selected subclasses of the $X$ states of various ranks for which the UWE (or a function of only $\Pi_{2}$ and $\Pi_{3}$ can be considered as an entanglement measure. In each case the UWE is proportional to a fourth-degree (or lower-degree) polynomial of $N$ or $C$. For the states given in cases $1, \ldots, 4$, and 8 , the witness $W$ is a good measure of entanglement because it is a function of $N$ with constant coefficients. The other states depend on an additional variable. These states include: the degenerate amplitude-damped states (case 5), rank-3 Bell-diagonal states (case 6), and pure states with isotropic noise (case 7). For these states, by measuring $\hat{W}$ does not provide enough information to determine the entanglement measures. However, for cases 1, 2, 3, and 8 listed in Table I, it is possible to determine $C$ and $N$ by measuring solely $\Pi_{2}$ and $\Pi_{3}$. The states of the largest and smallest ranks are the boundary states for $N$ versus $C$. The results are also visualized in Fig. 3 .

Note that we focus only on the states that depend on at most three independent variables. This is because, by allowing more freedom, we would have to measure all the first four moments of $\hat{\rho}^{\Gamma}$ to estimate the entanglement. This would give us no benefit with respect to the approach presented in the previous section. The states presented in Table I may appear rather specific. Note that $X$ states must be described, in general, by nine parameters (see, e.g., Ref. 82]). However, these states represent an infinite set of states that can be generated by local unitary transformations that do not change the entanglement. In other words, by applying local unitary operations, we can always obtain the following rank-specific real $X$ states [82]:

$$
\begin{aligned}
\hat{\rho}_{1} \equiv & \phi^{+}\left(\theta_{1}\right) \\
\hat{\rho}_{2 a} \equiv & p_{1} \phi^{+}\left(\theta_{1}\right)+p_{3} \psi^{+}\left(\theta_{3}\right) \\
\hat{\rho}_{2 b} \equiv & p_{1} \phi^{+}\left(\theta_{1}\right)+p_{2} \phi^{-}\left(\theta_{2}\right) \\
\hat{\rho}_{3} \equiv & p_{1} \phi^{+}\left(\theta_{1}\right)+p_{2} \phi^{-}\left(\theta_{2}\right)+p_{3} \psi^{+}\left(\theta_{3}\right) \\
\hat{\rho}_{4} \equiv & p_{1} \phi^{+}\left(\theta_{1}\right)+p_{2} \phi^{-}\left(\theta_{2}\right)+p_{3} \phi^{+}\left(\theta_{3}\right) \\
& +p_{4} \psi^{-}\left(\theta_{4}\right)
\end{aligned}
$$

which are incoherent mixtures of pure states

$$
\phi_{ \pm}(\theta)=\left(\begin{array}{cccc}
\cos ^{2} \theta & 0 & 0 & \pm \frac{1}{2} \sin (2 \theta) \\
0 & 0 & 0 & 0 \\
0 & 0 & 0 & 0 \\
\pm \frac{1}{2} \sin (2 \theta) & 0 & 0 & \sin ^{2} \theta
\end{array}\right)
$$

and

$$
\psi_{ \pm}(\theta)=\left(\begin{array}{cccc}
0 & 0 & 0 & 0 \\
0 & \cos ^{2} \theta & \pm \frac{1}{2} \sin (2 \theta) & 0 \\
0 & \pm \frac{1}{2} \sin (2 \theta) & \sin ^{2} \theta & 0 \\
0 & 0 & 0 & 0
\end{array}\right)
$$

with weights $p_{i}>0\left(\sum_{i} p_{i}=1\right)$. Note that the states, given in Eqs. (25) and (26), reduce to the Bell states for $\theta=\pi / 4$. One should be careful not to accidently reduce the rank of a given state by choosing some specific values of $\theta$. The relation between the $X$ states from Table $\Pi$ and the states defined in Eq. (24) is presented in Table II.

\section{CONCLUSIONS}

We have described a direct operational method for determining the negativity of an arbitrary two-qubit state. We have derived the method by analyzing the relation between the purity, negativity, and a universal entanglement witness for two-qubit entanglement. In particular, we have expressed the negativity as a function of six invariants which are linear combinations of nine from the complete set 21 fundamental and independent two-qubit invariants listed, e.g., in Ref. 64].

We have demonstrated how to measure the negativity of a two-photon polarization state by measuring three experimentally-accessible moments $\Pi_{n}$ of the partiallytransposed density matrix of a two-photon state. We pointed out that this approach can be more practical than directly estimating the negativity, which is sensitive even to a low-level noise.

We also discussed the possibility of using the universal entanglement witness or lower moments of $\hat{\rho}^{\Gamma}$ as a proper entanglement measure for some classes of states. In particular, we demonstrated their relation to the negativity and concurrence for the $X$ states.

It is worth noting that the UWE is not necessarily the least-error sensitive entanglement measure, which can be constructed from the moments of the partiallytransposed density matrix of a given state. It is possible that a better two-qubit entanglement measure exists that can be measured as a function of $\Pi_{n}$ for $n=2,3,4$.

We hope that these results can pave the way for direct and efficient methods for measuring two-qubit quantum entanglement.

\section{Acknowledgments}

We thank Paweł Horodecki for stimulating discussions. K. L. gratefully acknowledges the support by the Czech Science Foundation under the project No. 13-31000P. A.M. is supported by the Polish National Science Centre under grants DEC-2011/03/B/ST2/01903 and DEC2011/02/A/ST2/00305. K.B. acknowledges the support by the Foundation for Polish Science (START Programme) and the Polish National Science Centre under grant No. DEC-2013/11/D/ST2/02638, and the project No. LO1305 of the Ministry of Education, Youth and Sports of the Czech Republic. 
TABLE I: A survey on the relation between the concurrence $C$, negativity $N$, and universal entanglement witness $W$ for selected subclasses of the $X$ states, where $\vec{x}=(a, b, c, d, e)$ and $g_{n}=1-n f$. Note that $C$ and $W$ can be determined, in some cases, by measuring only $\Pi_{3}$ and (or) $\Pi_{2}$. However, in general, these entanglement measures can be obtained by measuring all the four moments of $\hat{\rho}^{\Gamma}$. The presented states of various ranks $R$ include: (Case 1) pure states, (2) rank-2 Bell-diagonal states, (3) phase-damped Bell states, (4) amplitude-damped pure states, (5) degenerate amplitude-damped states, (6) rank-3 Bell-diagonal states, (7) pure states with multimode noise, and (8) Werner states (rank-4 Bell-diagonal states). The moments $\Pi_{4}$ for all these eight subclasses of the $X$ states are given explicitly in Appendix A

\begin{tabular}{|c|c|c|c|c|c|c|}
\hline Case & $\hat{\rho}_{R}=\hat{\rho}(\vec{x})$ & $R$ & $\Pi_{2}$ & $\Pi_{3}$ & $W$ & $C$ \\
\hline 1 & $\begin{array}{l}a=b=f=0 \\
|d|=\sqrt{c e}\end{array}$ & 1 & 1 & $1-\frac{3}{4} N^{2}$ & $-\frac{N^{4}}{16}$ & $2|d|=N$ \\
\hline 2 & $\begin{array}{l}a=b=f<\frac{1}{2} \\
c=d=e=\frac{g_{2}}{2}\end{array}$ & $2 a$ & $\frac{1}{2}\left(N^{2}+1\right)$ & $\frac{1}{4}$ & $-\frac{N^{2}}{16}$ & $\left|g_{4}\right|=N$ \\
\hline 3 & $\begin{array}{l}a=b=f=0 \\
e=c=\frac{1}{2} \\
|d|<\frac{1}{2}\end{array}$ & $2 b$ & $\frac{1}{2}\left(N^{2}+1\right)$ & $\frac{1}{4}$ & $-\frac{N^{2}}{16}$ & $2|d|=N$ \\
\hline 4 & $\begin{array}{l}a=b=0 \\
f=1-c-e \\
|d|=\sqrt{c e}\end{array}$ & $2 b$ & $g_{2}+2 f^{2}$ & $\begin{array}{l}1-3\left(1+\frac{C^{2}}{2}\right) g_{1} \\
+3 g_{1}^{2}+\frac{3 C^{2}}{4}\end{array}$ & $-\frac{C^{4}}{16}$ & $\begin{array}{l}2|d| \\
=\sqrt{N^{2}+2 f N}\end{array}$ \\
\hline 5 & $\begin{array}{l}b=0 \\
a=f=\frac{1-c-e}{2} \\
|d|=\sqrt{c e}>f\end{array}$ & 3 & $g_{4}+6 f^{2}$ & $\begin{array}{l}g_{2}^{3}+2 f^{3} \\
-\frac{3}{4} g_{4}(C+2 f)^{2}\end{array}$ & $-\frac{C(C+4 f)(C+2 f)^{2}}{16}$ & $2|d|-2 f$ \\
\hline 6 & $\left\{\begin{array}{l}a=b=f<\frac{1}{4} \\
e=c=\frac{g_{2}}{2} \\
|d|<\sqrt{c e}\end{array}\right.$ & 3 & $\begin{array}{l}\frac{C}{8}(3 C+2) \\
+2|d|^{2}+\frac{3}{8}\end{array}$ & Eq. A2 & $-\frac{C\left(C^{2}+2 C+1-16|d|^{2}\right)}{64}$ & $\left|g_{4}\right|$ \\
\hline 7 & $\left\{\begin{array}{l}a=f<|d| \\
b=0 \\
c=c^{\prime}+\frac{f}{2} \\
e=e^{\prime}+\frac{f}{2} \\
|d|=\sqrt{c^{\prime} e^{\prime}}-\frac{f}{2}\end{array}\right.$ & 4 & $g_{3}^{2}+g_{3} f+\frac{5}{2} f^{2}$ & Eq. A3 & Eq. (A4) & $2|d|-2 f$ \\
\hline 8 & $\left\{\begin{array}{l}a=f<\frac{1}{6} \\
b=0 \\
c=e=\frac{g_{2}}{2} \\
|d|=\frac{g_{4}}{2}\end{array}\right.$ & 4 & $N+\frac{1}{3}(1-N)^{2}$ & $\begin{array}{l}\frac{1}{36}\left(-4 N^{3}+3 N^{2}\right. \\
+6 N+4)\end{array}$ & $\frac{1}{16}\left(\frac{1-N}{3}-1\right)^{3} N$ & $\left|g_{6}\right|=N$ \\
\hline
\end{tabular}

TABLE II: The relation between the states from Table $\$ and the $X$ states defined in Eq. (24). The correspondence is valid up to local unitary transformations on two qubits.

\begin{tabular}{ccccccccc}
\hline \hline Case & $p_{1}$ & $p_{2}$ & $p_{3}$ & $p_{4}$ & $\theta_{1}$ & $\theta_{2}$ & $\theta_{3}$ & $\theta_{4}$ \\
\hline 1 & 0 & 0 & 1 & 0 & 0 & 0 & $\operatorname{acos} \sqrt{c}$ & 0 \\
2 & $2 f$ & 0 & $g_{2}$ & 0 & $\frac{\pi}{4}$ & 0 & $\frac{\pi}{4}$ & 0 \\
3 & 0 & 0 & $\frac{1}{2}+|d|$ & $\frac{1}{2}-|d|$ & 0 & 0 & $\frac{\pi}{4}$ & $\frac{\pi}{4}$ \\
4 & $2 f$ & 0 & $g_{2}$ & 0 & $\frac{\pi}{2}$ & 0 & $\operatorname{acos} \sqrt{\frac{c}{g_{2}}}$ & 0 \\
5 & $f$ & $f$ & $g_{2}$ & 0 & $\frac{\pi}{4}$ & $\frac{\pi}{4}$ & $\operatorname{acos} \sqrt{\frac{c}{g_{2}}}$ & 0 \\
6 & $2 f$ & 0 & $g_{2}\left(\frac{1}{2}+|d|\right)$ & $g_{2}\left(\frac{1}{2}-|d|\right)$ & $\frac{\pi}{4}$ & 0 & $\frac{\pi}{4}$ & $\frac{\pi}{4}$ \\
7 & $f$ & $f$ & $g_{3}$ & $f$ & $\frac{\pi}{4}$ & $\frac{\pi}{4}$ & $\operatorname{acos} \sqrt{\frac{2 c-f}{2 g_{3}}}$ & $\frac{\pi}{4}$ \\
8 & $f$ & $f$ & $g_{3}$ & $f$ & $\frac{\pi}{4}$ & $\frac{\pi}{4}$ & $\frac{\pi}{4}$ & $\frac{\pi}{4}$ \\
\hline \hline
\end{tabular}

\section{Appendix A: Moments $\Pi_{3}$ and $\Pi_{4}$ for some states in} Table I

Here we show explicitly $\operatorname{det} \hat{\rho}^{\Gamma}$ and the moments $\Pi_{3}$ and $\Pi_{4}$ of the partially-transposed density matrix $\hat{\rho}^{\Gamma}$ for the selected subclasses of the $X$ states given in Table [. These moments are given as a function of either the concurrence $C$ or the negativity $N$.

The moments $\Pi_{4}^{(n)}$ for the $n$th case (subclass) of the 

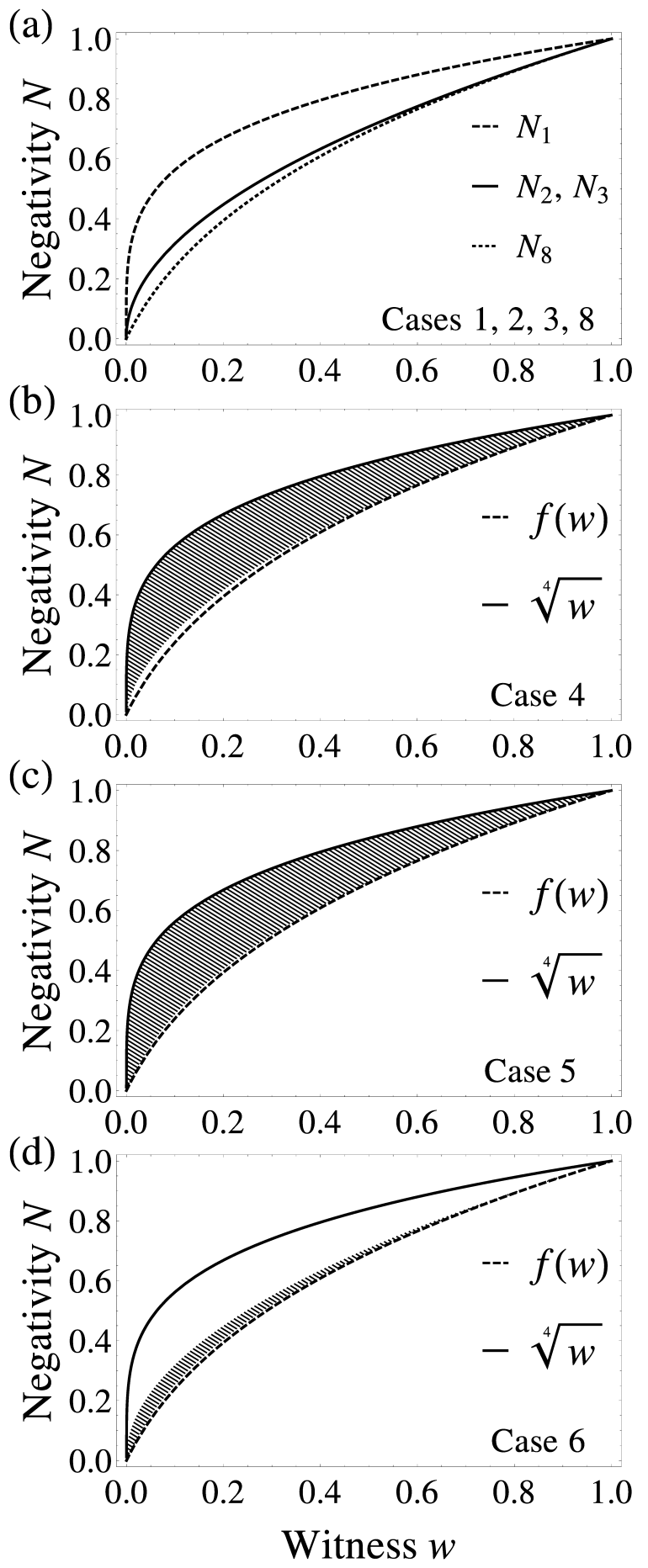

FIG. 3: Relation between the negativity $N$ and the universal entanglement witness $w$ for various states as defined in Table I In panel (a) we demonstrate the relations $N_{n}(w)$ for states, given in the $n$th case in Table $\mathrm{I}(\mathrm{b}),(\mathrm{c})$, and (d). The shaded areas depict the relation $N_{n}(w)$ for two-parameter states given in the cases for $n=4,5,6$, respectively. The covered area lies between the dashed curve corresponding to the lower bound $f(w)$, defined in Eq. $[16$, and the solid curve corresponding to the upper bound $\sqrt[4]{w}$. In all these panels, the shaded areas do not cover the whole space between the boundaries. The whole area is covered only in case 7 which, for brevity, is not presented here. Strictly speaking, the whole area is not covered in panel (c).
$X$ states analyzed in Table I are the following:

$$
\begin{aligned}
\Pi_{4}^{(1)}= & \left(1-\frac{N^{2}}{2}\right)^{2}, \\
\Pi_{4}^{(2)}= & \Pi_{4}^{(3)}=\frac{1}{8}\left(N^{4}+1\right), \\
\Pi_{4}^{(4)}= & \frac{C^{4}}{4}-g_{2} C^{2}+g_{1}^{4}+f^{4}, \\
\Pi_{4}^{(5)}= & {\left[g_{2}^{2}-\frac{1}{2}(C+2 f)^{2}\right]^{2}+3\left(C f+2 f^{2}\right)^{2}+2 f^{4}, } \\
\Pi_{4}^{(6)}= & 2^{-7}\left[9 C^{4}+4 C^{3}+6\left(2^{4} d^{2}+1\right) C^{2}\right. \\
& \left.+4\left(48 d^{2}+1\right) C+2^{8} d^{4}+96 d^{2}+9\right], \\
\Pi_{4}^{(7)}= & -\frac{3}{4} C^{2} g_{4}-\frac{3}{2} f^{3}-3 f g_{4} C+\frac{63}{4} f^{2}-\frac{15}{2} f+1, \\
\Pi_{4}^{(8)}= & \frac{1}{108}\left(7 N^{4}+2 N^{3}+6 N^{2}+8 N+4\right) .
\end{aligned}
$$

where $g_{n}=1-n f$, while $d$ and $f$ are the elements of $\hat{\rho}$, given in Eq. (18). The moment $\Pi_{3}$ for the $X$ state in case 6 reads

$$
\Pi_{3}=\frac{1}{32}\left[3 C\left(1-C^{2}+C+16|d|^{2}\right)+48|d|^{2}+5\right] .
$$

The moment $\Pi_{3}$ and $\operatorname{det} \hat{\rho}^{\Gamma}$ for the $X$ states in case 7 read

$$
\begin{aligned}
& \Pi_{3}=\frac{1}{4} C^{4}+2 f C^{3}+\frac{3}{4}\left(5 f^{2}-6 f-\frac{4}{3}\right) C^{2}+\frac{289}{8} f^{4} \\
& +f\left(f^{2}-18 f+4\right) C-\frac{89}{2} f^{3}+\frac{67}{2} f^{2}+g_{10},( \\
& \operatorname{det} \hat{\rho}^{\Gamma}=-\frac{1}{16} C^{4}-\frac{1}{2} f C^{3}-\frac{1}{16} f(15 f+2) C^{2} \\
& -\frac{1}{4} f^{2}(2-f) C \text {. }
\end{aligned}
$$

\section{Appendix B: Violation of the LOCC condition}

Here we show that the LOCC criterion C4., characterizing a good entanglement measure, can be violated for the UWE. Thus, we analyze the following two-qubit Bell-diagonal state

$$
\hat{\rho}=p \psi_{-}\left(\frac{\pi}{4}\right)+(1-p) \phi_{+}\left(\frac{\pi}{4}\right),
$$

for which $w(\hat{\rho})$ can increase under some local operations, as shown explicitly below.

As an example of an LOCC operation, we apply the "twirling" operation [83, where a random $\mathrm{SU}(2)$ rotation is performed on each qubit. This twirling changes $\hat{\rho}$ into the Werner state

$$
\begin{aligned}
\hat{\rho}^{\prime} & =p \psi_{-}\left(\frac{\pi}{4}\right)+\frac{1}{3}(1-p)\left[\phi_{+}\left(\frac{\pi}{4}\right)+\phi_{-}\left(\frac{\pi}{4}\right)+\psi_{+}\left(\frac{\pi}{4}\right)\right] \\
& =q \psi_{-}\left(\frac{\pi}{4}\right)+\frac{1}{4}(1-q) I,
\end{aligned}
$$

which is a mixture of the singlet state $\psi_{-}\left(\frac{\pi}{4}\right)$, with the weight $q=(4 p-1) / 3$, and the maximally-mixed state as given by the four-dimensional identity operator $I$. Consequently, for $p=(3 \sqrt{17}-7) / 8$, we observe the largest violation of the LOCC condition for this particular state $\hat{\rho}$. This is because, $w(\hat{\rho})=0.11719$ and $w\left(\hat{\rho}^{\prime}\right)=0.16294$, hence $w(\hat{\rho})<w\left(\hat{\rho}^{\prime}\right)$. 
It is worth noting that if these twirling operations are applied to the concurrence, negativity, or the REE, then property C.4 is always satisfied. Anyway, the twirling operations can be used to show that the Werner states determine the lower bounds of the concurrence for a given value of the negativity [51, the REE vs negativity [53, 84], or the REE vs the Bell nonlocality [50].

\section{Appendix C: Violation of the convexity condition}

Here we show that the convexity criterion C5., which is another important condition for a good entanglement measure, can also be violated for the UWE and some states.

Thus, let us consider a mixture $\hat{\rho}=\left(\hat{\rho}_{1}+\hat{\rho}_{2}\right) / 2$ of the following two-qubit density matrices

$$
\begin{aligned}
& \hat{\rho}_{1}=\frac{1}{2}\left[\phi_{+}(0)+\psi_{+}\left(\frac{\pi}{8}\right)\right], \\
& \hat{\rho}_{2}=\frac{1}{2}\left[\phi_{+}(0)+\psi_{-}\left(\frac{5 \pi}{8}\right)\right] .
\end{aligned}
$$

For these states, the convexity condition should imply that

$$
w(\hat{\rho}) \leq \frac{1}{2} w\left(\hat{\rho}_{1}\right)+\frac{1}{2} w\left(\hat{\rho}_{2}\right)
$$

However, the relevant values of the UWE read $w\left(\hat{\rho}_{1}\right)=$ $2^{-6}, w\left(\hat{\rho}_{2}\right)=2^{-6}$, and $w(\hat{\rho})=2^{-5}$. It is seen that $w\left(\hat{\rho}_{1}\right)+w\left(\hat{\rho}_{2}\right)=w(\hat{\rho})$. Thus, the convexity condition C3 is clearly violated because $w(\hat{\rho}) \not \leq \frac{1}{2} w(\hat{\rho})$ for $w(\hat{\rho})=\frac{1}{32}$.
[1] E. Schrödinger, "Discussion of Probability Relations between Separated Systems," Proc. Camb. Phil. Soc. 31, 555 (1935).

[2] A. Einstein, N. Podolsky, and B. Rosen, "Can QuantumMechanical Description of Physical Reality Be Considered Complete?," Phys. Rev. 47, 777 (1935).

[3] I. Bengtsson and K. Życzkowski, Geometry of Quantum States (Cambridge University Press, Cambridge, 2006).

[4] R. Horodecki, P. Horodecki, M. Horodecki, and K. Horodecki, "Quantum entanglement," Rev. Mod. Phys. 81, 865 (2009).

[5] W. P. Schleich and H. Walther, Elements of Quantum Information (Wiley-VCH, Weinheim, 2007).

[6] O. Krueger and R.F. Werner (eds.), "Some Open Problems in Quantum Information Theory", e-print quant$\mathrm{ph} / 0504166$.

[7] O. Gühne and G. Tóth, "Entanglement detection," Phys. Rep. 474, 1 (2009).

[8] M.G.A. Paris and J. Řeháček (eds.), Quantum State Estimation, Lecture Notes in Physics, Vol. 649 (Springer, Berlin, 2004).

[9] A. Miranowicz, K. Bartkiewicz, J. Perina Jr., M. Koashi, N. Imoto, and F. Nori, "Optimal two-qubit tomography based on local and global measurements", Phys. Rev. A 90, 062123 (2014).

[10] M. Horodecki, P. Horodecki, and R. Horodecki, "Separability of mixed states: necessary and sufficient conditions," Phys. Lett. A 223, 1 (1996).

[11] M. Bourennane et al., "Experimental Detection of Multipartite Entanglement using Witness Operators," Phys. Rev. Lett. 92, 087902 (2004).

[12] H. Häffner et al., "Scalable multiparticle entanglement of trapped ions," Nature (London) 438, 643 (2005).

[13] D. Leibfried et al., "Creation of a six-atom 'Schrödinger cat' state," Nature (London) 438, 639 (2005).

[14] F. A. Bovino, G. Castagnoli, A. Ekert, P. Horodecki, C. Moura Alves, and A. V. Sergienko, "Direct Measurement of Nonlinear Properties of Bipartite Quantum States," Phys. Rev. Lett. 95, 240407 (2005).

[15] P. Horodecki and A. Ekert, "Method for Direct Detection of Quantum Entanglement," Phys. Rev. Lett. 89, 127902 (2002).
[16] P. Horodecki, "From limits of quantum operations to multicopy entanglement witnesses and state-spectrum estimation," Phys. Rev. A 68, 052101 (2003).

[17] H. A. Carteret, "Noiseless Quantum Circuits for the Peres Separability Criterion," Phys. Rev. Lett. 94, 040502 (2005); "Exact interferometers for the concurrence and residual 3-tangle," quant-ph/0309212

[18] L. Aolita and F. Mintert, "Measuring Multipartite Concurrence with a Single Factorizable Observable," Phys. Rev. Lett. 97, 050501 (2006).

[19] S. P. Walborn, P. H. Souto Ribeiro, L. Davidovich, F. Mintert, and A. Buchleitner, "Experimental determination of entanglement with a single measurement," Nature (London) 440, 1022 (2006). L. Zhou and Y.-B. Sheng, Detection of nonlocal atomic entanglement assisted by single photons, Phys. Rev. A 90, 024301 (2014).

[20] P. Badziag, C. Brukner, W. Laskowski, T. Paterek, and M. Żukowski, "Experimentally Friendly Geometrical Criteria for Entanglement," Phys. Rev. Lett. 100, 140403 (2008).

[21] H. S. Park, S. S. B. Lee, H. Kim, S. K. Choi, and H. S. Sim, "Construction of an Optimal Witness for Unknown Two-Qubit Entanglement," Phys. Rev. Lett. 105, 230404 (2010).

[22] W. Laskowski, D. Richard, C. Schwemmer, T. Paterek, and H. Weinfurter, "Experimental Schmidt Decomposition and State Independent Entanglement Detection," Phys. Rev. Lett. 108, 240501 (2012).

[23] M. Huber, F. Mintert, A. Gabriel, and B. C. Hiesmayr, "Detection of High-Dimensional Genuine Multipartite Entanglement of Mixed States," Phys. Rev. Lett. 104, 210501 (2010).

[24] B. Jungnitsch, T. Moroder, and O. Gühne, "Taming multiparticle entanglement," Phys. Rev. Lett. 106, 190502 (2011).

[25] Ł. Rudnicki, P. Horodecki, and K. Życzkowski, "Collective Uncertainty Entanglement Test," Phys. Rev. Lett. 107, 150502 (2011).

[26] E. Rudnicki, Z. Puchała, P. Horodecki, and K. Życzkowski "Collectibility for mixed quantum states," Phys. Rev. A 86, 062329 (2012).

[27] Ł. Rudnicki, Z. Puchała, P. Horodecki, and K. Życz- 
kowski, "Constructive entanglement test from triangle inequality," J. Phys. A: Math. Theor. 47, 424035 (2014).

[28] A. Osterloh and P. Hyllus, "Estimating multipartite entanglement measures," Phys. Rev. A 81, 022307 (2010).

[29] G. Puentes, A. Datta, A. Feito, J. Eisert, M. B. Plenio, and I. A. Walmsley, "Entanglement quantification from incomplete measurements: Applications using photonnumber-resolving weak homodyne detectors," New J. Phys. 12, 033042 (2010).

[30] J. Jurkowski and D. Chruściński, "Estimating concurrence via entanglement witnesses," Phys. Rev. A 81, 052308 (2010).

[31] Y.C. Liang, T. Vértesi, and N. Brunner, "Semi-deviceindependent bounds on entanglement," Phys. Rev. A 83 022108 (2011).

[32] P. Silvi, F. Taddei, R. Fazio, and V. Giovannetti, "Quantitative entanglement witnesses of isotropic and Werner classes via local measurements," J. Phys. A: Math. Theor. 44, 145303 (2011).

[33] S.S. B. Lee and H.S. Sim, "Quantifying mixed-state quantum entanglement by optimal entanglement witnesses," Phys. Rev. A 85022325 (2012).

[34] A. Osterloh and J. Siewert, "Invariant-based entanglement monotones as expectation values and their experimental detection," Phys. Rev. A 86042302 (2012).

[35] S. Ryu, S.S.B. Lee, and H.S. Sim, "Minimax optimization of entanglement witness operator for the quantification of three-qubit mixed-state entanglement," Phys. Rev. A 86, 042324 (2012).

[36] C. Zhang, S. Yu, Q. Chen, and C. H. Oh, "Detecting and Estimating Continuous-Variable Entanglement by Local Orthogonal Observables," Phys. Rev. Lett. 111, 190501 (2013).

[37] S. M. Hashemi Rafsanjani, C. J. Broadbent, and J. H. Eberly, "Bounding the entanglement of $N$ qubits with only four measurements," Phys. Rev. A 88, 062331 (2013).

[38] O. Gühne, M. Reimpell, and R.F. Werner, "Estimating entanglement measures in experiments," Phys. Rev. Lett. 98, 110502 (2007).

[39] J. Eisert, F. Brandao, and K. Audenaert, "Quantitative entanglement witnesses," New J. Phys. 9, 46 (2007).

[40] O. Gühne, M. Reimpell, R.F. Werner, "Lower bounds on entanglement measures from incomplete information," Phys. Rev. A 77, 052317 (2008).

[41] F.G.S.L. Brandao, "Quantifying entanglement with witness operators," Phys. Rev. A 72, 022310 (2005).

[42] H.-P. Breuer, "Separability criteria and bounds for entanglement measures," J. Phys. A: Math. Gen. 39, 11847 (2006).

[43] K.M.R. Audenaert and M.B. Plenio, "When are correlations quantum? Verification and quantification of entanglement by simple measurements," New J. Phys. 8, 266 (2006).

[44] F. Mintert, "Concurrence via entanglement witnesses," Phys. Rev. A 75, 052302 (2007).

[45] A. Datta, S. Flammia, A. Shaji, and C. Caves, "Constrained bounds on measures of entanglement," Phys. Rev. A 75, 062117 (2007).

[46] M. Genoni, P. Giorda, and M. Paris, "Optimal estimation of entanglement," Phys. Rev. A 78, 032303 (2008).

[47] Z.-H. Chen, Z.-H. Ma, O. Gühne, and S. Severini, "Estimating Entanglement Monotones with a Generalization of the Wootters Formula," Phys. Rev. Lett. 109200503
(2012).

[48] F. Verstraete and M.M. Wolf, "Entanglement versus Bell violations and their behavior under local filtering operations," Phys. Rev. Lett. 89, 170401 (2002).

[49] K. Bartkiewicz, K. Lemr, B. Horst, and A. Miranowicz, "Entanglement estimation from Bell inequality violation," Phys. Rev. A 88, 052105 (2013).

[50] B. Horst, K. Bartkiewicz, and A. Miranowicz, "Two-qubit mixed states more entangled than pure states: Comparison of the relative entropy of entanglement for a given nonlocality," Phys. Rev. A 87, 042108 (2013).

[51] F. Verstraete, K. M. R. Audenaert, J. Dehaene, and B. De Moor, "A comparison of the entanglement measures negativity and concurrence," J. Phys. A 34, 10327 (2001).

[52] A. Miranowicz and A. Grudka, "Ordering two-qubit states with concurrence and negativity," Phys. Rev. A 70, 032326 (2004);

[53] A. Miranowicz and A. Grudka, "A comparative study of relative entropy of entanglement, concurrence and negativity," J. Opt. B 6, 542 (2004).

[54] A. Miranowicz, S. Ishizaka, B. Horst, and A. Grudka, "Comparison of the relative entropy of entanglement and negativity," Phys. Rev. A 78, 052308 (2008).

[55] R. Augusiak, M. Demianowicz, and P. Horodecki, "Universal observable detecting all two-qubit entanglement and determinant-based separability tests," Phys. Rev. A 77, 030301 (2008).

[56] K. Bartkiewicz, P. Horodecki, K. Lemr, A. Miranowicz, and K. Życzkowski, "Method for universal detection of two-photon polarization entanglement," e-print arXiv:1405.5560 1 .

[57] K. Bartkiewicz, K. Lemr, A. Černoch, and J. Soubusta, "Measuring nonclassical correlations of two-photon states," Phys. Rev. A 87, 062102 (2013).

[58] M. Bula, K. Bartkiewicz, A. Cernoch, and K. Lemr, "Entanglement-assisted scheme for nondemolition detection of the presence of a single photon," Phys. Rev. A 87, 033826 (2013); E. Meyer-Scott, M. Bula, K. Bartkiewicz, A. Cernoch, J. Soubusta, T. Jennewein, and K. Lemr "Entanglement-based linear-optical qubit amplifier," Phys. Rev. A 88, 012327 (2013).

[59] K. Bartkiewicz, K. Lemr, and A. Miranowicz, "Direct method for measuring of purity, superfidelity, and subfidelity of photonic two-qubit mixed states," Phys. Rev. A 88, 052104 (2013).

[60] A. Peres, "Separability Criterion for Density Matrices," Phys. Rev. Lett. 77, 1413 (1996).

[61] M. Bartkowiak, A. Miranowicz, X. Wang, Y.X. Liu, W. Leonski, and F. Nori, "Sudden vanishing and reappearance of nonclassical effects: General occurrence of finitetime decays and periodic vanishings of nonclassicality and entanglement witnesses," Phys. Rev. A 83, 053814 (2011).

[62] F. Mintert, "Entanglement measures as physical observables," Appl. Phys. B 89, 493 (2007).

[63] Y. Makhlin, "Nonlocal properties of two-qubit gates and mixed states and optimization of quantum computations," Quantum Inf. Process. 1(4), 243 (2002).

[64] R. King and T. Welsh, "Qubits and invariant theory," J. Phys.: Conf. Ser. 30, 1 (2006).

[65] K. Życzkowski, P. Horodecki, A. Sanpera, and M. Lewenstein, "Volume of the set of separable states," Phys. Rev. A 58, 883 (1998).

[66] G. Vidal and R. F. Werner, "Computable measure of en- 
tanglement," Phys. Rev. A 65, 032314 (2002).

[67] K. Audenaert, M. B. Plenio, and J. Eisert, "Entanglement Cost under Positive-Partial-Transpose-Preserving Operations," Phys. Rev. Lett. 90, 027901 (2003).

[68] S. Ishizaka, "Binegativity and geometry of entangled states in two qubits," Phys. Rev. A 69, 020301(R) (2004).

[69] C. Eltschka and J. Siewert, "Negativity as an Estimator of Entanglement Dimension," Phys. Rev. Lett. 111, 100503 (2013).

[70] S. Rana, "Negative eigenvalues of partial transposition of arbitrary bipartite states," Phys. Rev. A 87, 054301 (2013); A. Sanpera, R. Tarrach, and G. Vidal, "Local description of quantum inseparability," ibid. 58, 826 (1998).

[71] W. K. Wootters, "Entanglement of Formation of an Arbitrary State of Two Qubits," Phys. Rev. Lett. 80, 2245 (1998).

[72] M. Sinołęcka, K. Życzkowski, and M. Kuś, "Manifolds of equal entanglement for composite quantum systems," Acta Phys. Pol. B 33, 2081 (2002); F. Verstraete, J. Dehaene, and B. De Moor, "Normal forms and entanglement measures for multipartite quantum states," Phys. Rev. A 68, 012103 (2003); G. Gour, "Family of concurrence monotones and its applications," ibid. 71, 012318 (2005).

[73] R.F. Werner, "Quantum states with Einstein-PodolskyRosen correlations admitting a hidden-variable model," Phys. Rev. A 40, 4277 (1989).

[74] P.E.M.F. Mendonca, M.A. Marchiolli, and D. Galetti, "Entanglement universality of two-qubit X-states," eprint arXiv:1407.3021.

[75] G. Rigolin, "Thermal entanglement in the two-qubit Heisenberg XYZ model," Int. J. Quant. Inf. 2, 393 (2004).

[76] T. Chen, Y.-X. Huang, C.-J. Shan, J.-X. Li, J.-B. Liu, and T.-K. Liu, "Entanglement evolution in an anisotropic two-qubit Heisenberg XYZ model with DzyaloshinskiiMoriya interaction," Chin. Phys. B 19, 050302 (2010).

[77] T. Yu and J. H. Eberly, "Finite-Time Disentanglement Via Spontaneous Emission," Phys. Rev. Lett. 93, 140404 (2004).

[78] Z. Ficek and R. Tanaś, "Dark periods and revivals of entanglement in a two-qubit system," Phys. Rev. A 74, 024304 (2006); "Delayed sudden birth of entanglement," ibid. 77, 054301 (2008).

[79] M. Demianowicz, "Reexamination of determinant-based separability test for two qubits," Phys. Rev. A 83, 034301 (2011).

[80] T. Yu and J.H. Eberly, "Evolution from entanglement to decoherence of bipartite mixed X states," Quant. Inf. Comp. 7, 459 (2007).

[81] A. Miranowicz, "Violation of Bell inequality and entanglement of decaying Werner states," Phys. Lett. A 327, 272 (2004).

[82] S.R. Hedemann, "Evidence that All States Are Unitarily Equivalent to X States of the Same Entanglement," eprint arXiv:1310.7038 4 .

[83] C. H. Bennett, G. Brassard, S. Popescu, B. Schumacher, J. A. Smolin, and W. K. Wootters, "Purification of Noisy Entanglement and Faithful Teleportation via Noisy Channels," Phys. Rev. Lett. 76, 722 (1996); C. H. Bennett, D. P. DiVincenzo, J. A. Smolin, and W. K. Wootters, "Mixed-state entanglement and quantum error correction," Phys. Rev. A 54, 3824 (1996).

[84] V. Vedral and M. B. Plenio, "Entanglement measures and purification procedures," Phys. Rev. A 57, 1619 (1998). 